\title{
Wireless Location Technology Of Gauss Particle Filter Under NLOS Environment
}

\author{
Linkai Yan ${ }^{1, a}$, Yongyi Mao ${ }^{2, b}$
}

1. School of Communication and Information Engineering, Xi'an University of Posts \& Telecommunications, Xi'an 710061, China;

\author{
2. School of Electronic Engineering, Xi'an University of Posts \& Telecommunications, Xi'an 710061, \\ China. \\ ayan_linkai@163.com, bmaoyongyi@263.net
}

Keywords: Wireless location, Non line of sight, Particle filter, Gauss transform, Time difference of arrive

\begin{abstract}
In cellular wireless location, the non line of sight (NLOS) error can seriously affect the accuracy of the position estimation of the mobile station. A Gaussian particle filter localization algorithm (Gauss-PF) is proposed, which is based on the Gauss transform. In this algorithm, the high precision of Gauss filtering and the latest observation data are used to ensure the accuracy of the mobile station, which can not only reduce the NLOS error of the nonlinear filter, but also solve the problem of particle degradation in classical particle filter. The simulation results show that the algorithm can maintain high accuracy and robustness in the case of NLOS error.
\end{abstract}

\section{Introduction}

In recent years, wireless positioning technology based on the cellular network has been widely used in many fields such as target tracking [1], image processing [2], and map navigation [3]. Cellular network of wireless location mainly includes the signal strength (SS) positioning method, the angle of arrivals (AOA) positioning method and the time difference of arrival (TDOA) positioning method, and the positioning method based on time difference of arrival become the most commonly used method due to the simple algorithm, high positioning accuracy. As a result, the CHAN algorithm [4] and the Taylor series expansion method [5] are proposed. On this basis a fusion algorithm [6] which combined with CHAN algorithm and the Taylor series expansion algorithm is presented. However, the algorithms apply only to non line-of-sight environments.

In nonlinear non-Gaussian environment, location accuracy will be serious decline due to the complexity of noise and multipath interference. In order to reduce the error, a method of un-scent Kalman filtering is proposed [7]. The algorithm improves the accuracy by approximation of nonlinear model, but it does not apply to non-Gaussian environment. A method which based on un-scent particle filter for target tracking is proposed in [8] and it has achieved a certain effect under nonlinear and non-Gaussian environment. The Gaussian particle filter algorithm is based on TDOA measurement information using Gaussian transform method.

\section{The nonlinear model and the particle filter algorithm}

Assume that the state equation and measurement equation of the nonlinear system can be described as follows,

$\mathbf{x}_{\mathbf{k}}=f\left(\mathbf{x}_{\mathbf{k}-1}\right)+\mathbf{e}_{\mathbf{k}-1}$

$\mathbf{y}_{\mathbf{k}}=h\left(\mathbf{x}_{\mathbf{k}}\right)+\mathbf{n}_{\mathbf{k}}$

Where $\mathbf{x}_{\mathbf{k}} \in \mathbf{R}^{\mathbf{n}}$ is the state vector in $\mathrm{k}$ time, $\mathbf{y}_{\mathbf{k}} \in \mathbf{R}^{\mathbf{n}}$ is the measurement vector in $\mathrm{k}$ time, $\mathbf{e}_{\mathbf{k}}$ is the state noise and $\mathbf{n}_{\mathbf{k}}$ is the measurement noise, $\mathbf{e}_{\mathbf{k}}$ and $\mathbf{n}_{\mathbf{k}}$ are independence each other and the noise sequence which the covariance is $\mathbf{Q}$ and $\mathbf{R}$.

The basic idea of the particle filter (PF) is, to find a set of random samples (particles) to 
represent the posterior probability function with the sample average instead of integral operation, thus the minimum variance estimation is obtained [9]. A detailed description as follows: Assume that the posterior probability density of system is $p\left(x_{k-1} \mid y_{k-1}\right)$ in k-1 time, and then select k random samples from sample space, after acquisition of measuring information in $\mathrm{k}$ time, after time and status updates, posterior probability density of particles can approximate $p\left(x_{k} \mid y_{k}\right)$. The PF algorithm can be shown as follows:

(1) Initialization

According to the prior probability density $p\left(x_{0} \mid y_{0}\right)$, constructing sampling particles is $\left\{x_{0}^{(i)}\right\}_{i=1}^{N}$.

The importance weights of initialized particles: $w_{0}^{i}=1 / N$;

(2) Importance sampling

Updated sampling particles $\left\{x_{k}^{(i)}\right\}_{i=1}^{N}$ from important density function $q\left(x_{k} \mid x_{0: k-1}^{(i)}, y_{1: k}\right)$,

Updated the importance weights of particles:

$w_{k}^{(i)}=w_{k-1}^{(i)} \frac{p\left(y_{k} \mid x_{k}^{(i)}\right) p\left(x_{k}^{(i)} \mid x_{k-1}^{(i)}\right)}{q\left(x_{k}^{(i)} \mid x_{0: k-1}^{(i)}, y_{1: k}\right)}$

Where, the important density function select optimal important density function $[10,11]$, then, $q\left(x_{k}^{(i)} \mid x_{0: k-1}^{(i)}, y_{1: k}\right)=p\left(x_{k}^{(i)} \mid x_{k-1}^{(i)}\right)$

Normalized weights can be expressed as:

$\widetilde{w}_{k}^{(i)}=w_{k}^{(i)} / \sum_{j=1}^{k} w_{k}^{(i)}$

(3) Re-sampling

In order to overcome particles degradation phenomenon, re-sampling is proposed. It is a process of removing those particles with lower importance weights and copying those particles with high importance weights [12]. Set the effective sampling scale be defined as:

$N_{\text {eff }}=1 / \sum_{i=1}^{N}\left(\widetilde{w}_{k}^{(i)}\right)^{2}$

If $N_{\text {eff }}<N_{t h}$, then re-sample. Where $N_{t h}$ is threshold.

(4) State estimation

$\hat{x}_{k}=\sum_{i=1}^{N} \widetilde{w}_{k}^{(i)} x_{k}^{(i)}$

Turn to step (2) and continue the operations.

Due to selecting prior density function instead of important density function without considering the latest observation data, result in the posterior probability distribution has a big error [13]. So this method is only suitable for estimation of lower precision.

\section{Gaussian particle filter algorithm}

\subsection{Gaussian filter}

Gauss integral formula is a Gaussian numerical integral, it can improve the algebraic accuracy by choose Gauss point. In one dimensional case, the integral formula can be shown as follows [14]

$I=\int f(x) W(x) d x \approx \sum_{i=1}^{m} w_{i} f\left(\xi_{i}\right)$

Where $W(x)$ is the weight function, $\xi_{i}$ are the Gauss points, $w_{i}$ are the corresponding weights and $\mathrm{m}$ is the coefficient of Gaussian point, When $\xi_{i}$ and $w_{i}$ choose corresponding Gauss points and weights respectively, on the type of no more than $2 \mathrm{~m}+1$ polynomial can accurately established for $f(x)[15,16]$. Setting the weight function as standard normal distribution, the integral formula can approximate 
$I=\int f(x) \frac{1}{\left((2 n)^{n} \operatorname{det}(\Sigma)\right)^{1 / 2}} \exp \left(-\frac{(x-\bar{x})^{T} \Sigma^{-1}(x-\bar{x})}{2}\right) d x \approx \sum_{i=1}^{m} w_{i} f\left(\xi_{i}\right)$

Where $\bar{x}$ and $\Sigma$ are the mean and the covariance respectively, the transformation formula between Gauss point is

$x_{i}=\sqrt{\Sigma}^{T} \xi_{i}+\bar{x}$

Assume that the system state and variance are $x_{k-1 \mid k-1}$ and $P_{k-1 \mid k-1}$ in k moment, the system state and variance can forecast next step by the means of Gauss point transformation formula $x_{i}={\sqrt{P_{k-1 \mid k-1}}}^{T} \xi_{i}+x_{k-1 \mid k-1}$ and the system state transition equation,

$x_{k \mid k-1}=\sum_{i=1}^{m} w_{i} f\left(x_{i}\right)$

$P_{k \mid k-1}=Q+\sum_{i=1}^{m} w_{i}\left(f\left(x_{i}\right)-x_{k \mid k-1}\right)\left(f\left(x_{i}\right)-x_{k \mid k-1}\right)^{T}$

On this basis of the above formula, the system state and variance can update by the means of the new Gauss point transformation formula $x_{i}={\sqrt{P_{k \mid k-1}}}^{T} \xi_{i}+x_{k \mid k-1}$ and the system state transition equation,

$X_{k \mid k}=X_{k \mid k-1}+K_{k}\left(y_{k}-z_{k}\right)$

$P_{k \mid k}=P_{k \mid k-1}-K_{k} P_{x z}^{T}$

Where,

$z_{k}=\sum_{i=1}^{m} w_{i} h\left(x_{i}\right)$

$P_{x z}=\sum_{i=1}^{m} w_{i}\left(x_{i}-x_{k \mid k-1}\right)\left(h\left(x_{i}\right)-z_{k}\right)^{T}$

$P_{z z}=\sum_{i}^{m} w_{i}\left(h\left(x_{i}\right)-z_{k}\right)\left(h\left(x_{i}\right)-z_{k}\right)^{T}$

$K_{k}=P_{x z}\left(R+P_{z z}\right)^{-1}$

\subsection{Gaussian particle filter algorithm}

Gaussian particle filter (Gauss-PF) algorithm assumes that the state of the system posterior probability function can be approximated by a Gaussian distribution. This algorithm takes into account the latest observations, it can better improve the filtering accuracy.

Particle filter algorithm based on Gauss filter algorithm can be described as follows:

(1) Initialization

Sampling particles $\left\{x_{0}^{(i)}\right\}_{i=1}^{N}$ from the prior probability density $p\left(x_{0}\right)$, then $x_{0} \sim p\left(x_{0}\right)$;

(2) Gaussian filtering

Conduct Gaussian filtering for a collection of sample $\left\{x_{k-1}^{(i)}\right\}_{i=1}^{N}$ in k-1 moment, getting the estimation of particles and its variance is $x_{k \mid k}^{(i)}$ and $P_{k \mid k}^{(i)}$, thus important density function can be expressed as

$$
q\left(x_{k}^{(i)} \mid x_{0: k-1}^{(i)}, y_{1: k}\right)=N\left(x_{k \mid k}^{(i)}, P_{k \mid k}^{(i)}\right)
$$

(3) Importance sampling

Sampling particles $\left\{x_{k}^{(i)}\right\}_{i=1}^{N}$ from the importance density function $N\left(x_{k \mid k}^{(i)}, P_{k \mid k}^{(i)}\right)$, then $x_{k}^{(i)} \sim N\left(x_{k \mid k}^{(i)}, P_{k \mid k}^{(i)}\right)$

Calculate importance weights of the particles by formula (3).

Normalize the particle weights.

(4) Re-sampling 
Determine whether to re-sample as required. After re-sampling, the original sample set is mapped to the equal-weighted sample set: $\left\{x_{k}^{(i)}, \widetilde{w}_{k}^{(i)}\right\} \rightarrow\left\{\widetilde{x}_{k}^{(i)}, 1 / N\right\}$

(5) Particle state estimation

Turn to the second step and recursively calculate the state of the next moment.

\section{Simulation and analysis}

\subsection{Wireless positioning system model}

Considered in a cellular network environment, we establish the equations by three base stations (BS) with TDOA measurement information. The dynamic state space model is given by:

$$
\begin{aligned}
& \mathbf{x}_{\mathbf{k}}=\mathbf{A} \mathbf{x}_{\mathbf{k}-\mathbf{1}}+\mathbf{G e}_{\mathbf{k}-\mathbf{1}} \\
& =\left[\begin{array}{cccc}
1 & \Delta T & 0 & 0 \\
0 & 1 & 0 & 0 \\
0 & 0 & 1 & \Delta T \\
0 & 0 & 0 & 1
\end{array}\right]\left[\begin{array}{l}
x_{k-1} \\
\&_{k-1} \\
y_{k-1} \\
\&_{k-1}
\end{array}\right]+\left[\begin{array}{cc}
\Delta T^{2} / 2 & 0 \\
\Delta T & 0 \\
0 & \Delta T^{2} / 2 \\
0 & \Delta T
\end{array}\right]\left[\begin{array}{l}
e_{x} \\
e_{y}
\end{array}\right] \\
& =\left[\begin{array}{l}
\sqrt{\left(x_{k}-x_{1}\right)^{2}+\left(y_{k}-y_{1}\right)^{2}}-\sqrt{\left(x_{k}-x_{2}\right)^{2}+\left(y_{k}-y_{2}\right)^{2}} \\
\sqrt{\left(x_{k}-x_{1}\right)^{2}+\left(y_{k}-y_{1}\right)^{2}}-\sqrt{\left(x_{k}-x_{3}\right)^{2}+\left(y_{k}-y_{3}\right)^{2}}
\end{array}\right]+\left[\begin{array}{l}
n_{12} \\
n_{13}
\end{array}\right]
\end{aligned}
$$

Where, $\mathbf{x}_{\mathbf{k}}$ represents state information of the mobile station at $\mathrm{k}$ time, $\left[x_{k}, y_{k}\right]$ is the coordinates of the mobile station, [\&, \& $\left.\&_{k}\right]$ is the speed of the mobile station, $\Delta T$ represents the sampling interval, $\mathbf{e}_{\mathbf{k}}$ is the process noise sequence which is exponential distribution, $\left(x_{1}, y_{1}\right),\left(x_{2}, y_{2}\right),\left(x_{3}, y_{3}\right)$ represents the location of the three stations, $\mathbf{y}_{\mathbf{k}}$ represents TDOA measurements from the mobile station to the base station, $\mathbf{n}_{\mathbf{k}}$ is the measurement noise of Gaussian distribution.

\subsection{Simulation conditions}

The PF algorithm and Gauss - PF algorithm are applied to tracking in the cellular system. Relevant parameter are setting as follows: $\mathrm{BS} 1=(0,0), \mathrm{BS} 2=(0,100), \mathrm{BS} 3=(100 \sqrt{3}, 50)$, the initial position of mobile station is $(10,10)$, the speed of mobile station is $(2,4)$, the process noise $e_{k-1} \sim \operatorname{Exp}(2)$, the measurement noise $n_{k} \sim N\left(0,0.1^{2}\right), \Delta T=1 \mathrm{~s}$, the particle numbers is 100 , sampling time is $60 \mathrm{~s}$, the initial covariance $P_{0 \mid 0}=\operatorname{diag}\left(2,4^{2}, 3,5^{2}\right)$, the Gauss points $\mathrm{m}=3$, the mobile station tracking of PF and Gauss-PF is simulate in 50 Monte Carlo runs, the simulation are as follows,

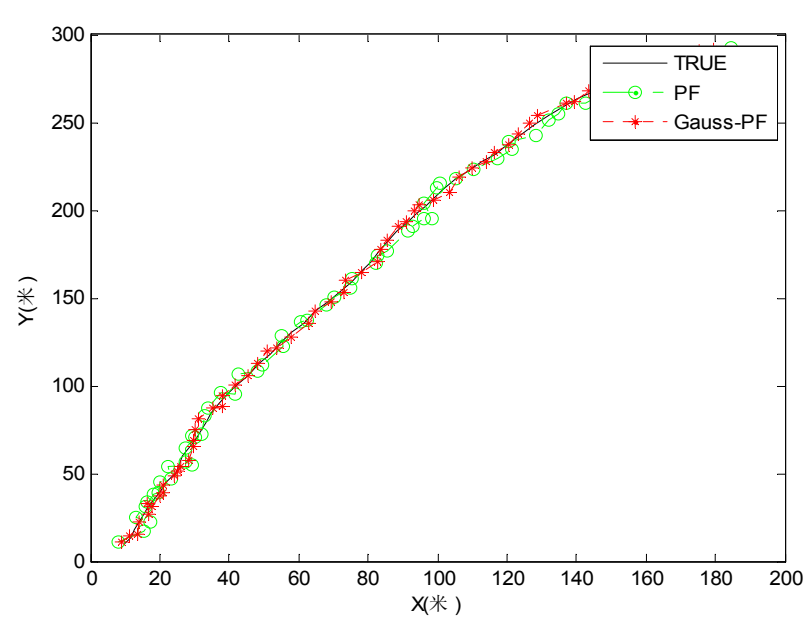

Fig 1 Tracking trajectory of the mobile station 


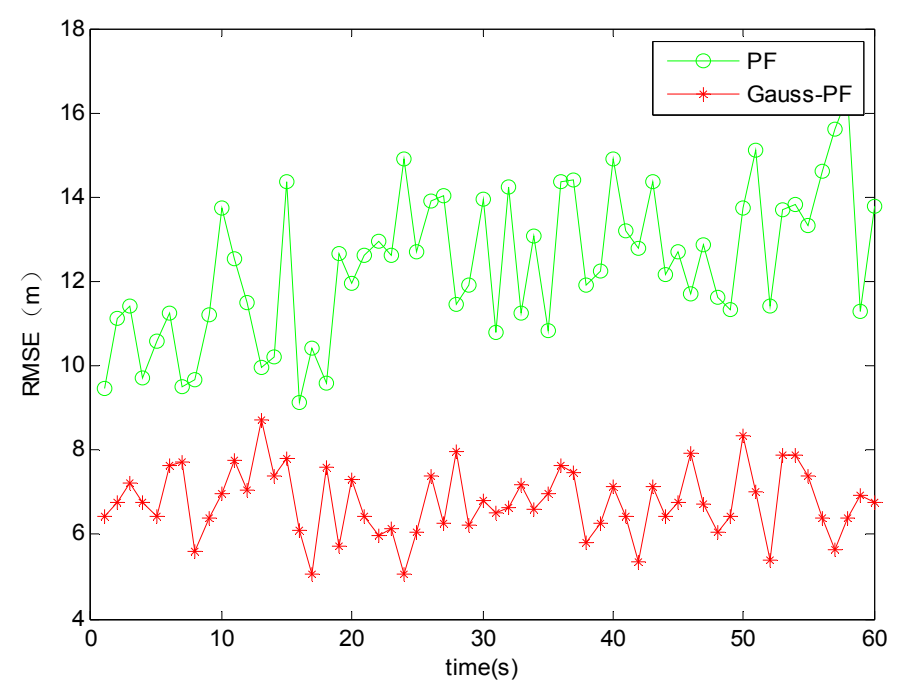

Fig 2 RMSE of the mobile station

The trajectory tracking of the mobile station is shown in Fig. 1.In the same conditions, the Gauss - PF is much closer to the real trajectory. It is obvious that Gauss-PF has much better estimation accuracy than PF in non-Gaussian environment.

In Fig. 2, we compare the RMSE performance of the mobile station when the tracking of the two algorithm in non-Gaussian environment. It is found that the RMSE of Gauss-PF algorithm has smaller fluctuations than PF algorithm. The result indicates that Gauss-PF can improve accuracy and reduce error.

\section{Summary}

The proposed Gauss-PF algorithm, which is based on Gaussian particle filter in cellular network, absorb the advantages of the Gaussian filtering and particle filtering, and obtain the better importance density function by using high precision Gaussian integral formula. The probability density function considers the latest observation, therefore it is closer to the posterior probability distribution of systems. The accuracy of mobile station is improved while solve particle degradation problem. In an actual positioning and tracking in cellular network, noise are usually non-Gaussian model, and the Gauss-PF algorithm still has better estimation precision under non-Gaussian environment. Compared with the particle filter algorithm, the proposed algorithm can effectively improve the accuracy of the mobile station and reduce the NLOS error.

\section{References}

[1] K Y Ng, K Y Lam, J C Cheng, et al. An effective signal strength-based wireless location estimation system for tracking indoor mobile users. Journal of Computer \& System Sciences. Vol. 79 (2013) No. 7, p. 1005-1016.

[2] A Arafa, X Jin, M H Bergen, et al. Characterization of image receivers for optical wireless location technology. IEEE Photonics Technology Letters. Vol. 27 (2015) No.18, p. 1923-1926.

[3] J P Zhou, L W Liu, G F Liao, et al. Predictive and fault-tolerant location service in mobile Ad Hoc networks. Wireless Personal Communication. Vol.71 (2013) No. 4, p. 3115-3130.

[4] J W Zhang, C L Yu, Y Y Ji. The performance analysis of Chan location algorithm in cellular network. World Congress on Computer Science \& Information Engineering. Los Angeles, USA, March 31-April 2, 2009, p. 339-343.

[5] L F Peng, Q Z Huang, Y S Lin. A cooperative localization method based on conjugate gradient and Taylor series expansion algorithms. International Conference on Computer Science and Network Technology. Harbin, China, December 24-26, 2011, p. 1108-1112. 
[6] D Z Chen, H Tang, J D Wu. Research of TDOA cooperative location algorithm based on Chan and Taylor. Computer Science. Vol. 38 (2011) No. 10, p. 406-411.

[7] J Y Zuo, Y N Jia, Q X Gao. Simplified unscented particle filter for nonlinear/non-Gaussian Bayesian estimation. Systems Engineering and Electronics. Vol. 24 (2013) No. 3, p. 537-544.

[8] X Z Zhao, S M Zhang. Distributed strong tracking unscented particle filter for simultaneous localization and mapping. The 33rd Chinese Control Conference. Nanjing, China, July 28-30, 2014, p. 978-983.

[9] C Cheng, R Ansari. Kernel particle filter for visual tracking. IEEE Signal Processing Letters. Vol. 12 (2005) No.3, p. 242-245.

[10]S W Fa, J L Yue. Improving particle filter with a new sampling strategy. Computer Science \& Technology. Vol. 140 (2009) No.2, p. 107-113.

[11]S Saikat, G Fredrik. Particle filtering with dependent noise processes. IEEE Trans on Signal Processing. Vol. 60 (2012) No.9, p. 4479-4508.

[12]C Gonzales, S Dubuisson. Combinatorial resampling particle: an effective and efficient method for articulated object tracking. International Journal of Computer Vision. Vol. 112 (2014) No.3, p. 255-284.

[13]F Gustafsson. Particle filter theory and practice with positioning applications. IEEE Aerospace \& Electronic Systems Magazine. Vol. 25 (2010) No.7, p. 53-58.

[14]I Kazufumi, K Q Xiong. Gaussian filter for nonlinear filtering problems. IEEE Trans on Automatic Control. Vol. 45 (2000) No.5, p. 910-927.

[15]L Jaechan, H Daehyoung. Gaussian particle filtering approach for carrier frequency offset estimation in OFDM systems. Signal Processing Letters. Vol. 20 (2013) No.4, p. 367-370.

[16]J L Yang, H B Ji, J M Liu. Gauss-Hermite particle PHD filter for bearings-only multi-target tracking. Systems Engineering \& Electronics. Vol.35 (2013) No.3, p. 457-462. 